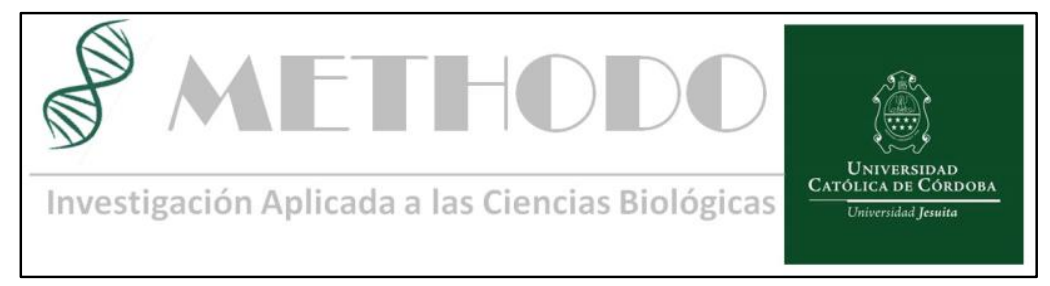

\title{
Nanotecnología en Odontología: Aspectos generales y posibles aplicaciones.
}

\section{Nanotechnology in Dentistry: General aspects and potentialapplications.}

\author{
Gustavo F. Molina, Prof. Dr ${ }^{1}$, Santiago D. Palma, Prof. Dr. ${ }^{2}$ \\ ${ }^{1}$ Profesor Titular de la Cátedra de Materiales Dentales y Biomateriales, Carrera de Odontología, Facultad de Ciencias de la Salud, \\ Universidad Católica de Córdoba \\ ${ }^{2}$ Profesor Asociado del Departamento de Ciencias Farmacéuticas, Facultad de Ciencias Químicas, Universidad Nacional de \\ Córdoba. Investigador Principal de CONICET
}

\section{Resumen:}

Las innovaciones tecnológicas en Odontología procuran desde las Nanociencias dar solución a una diversidad de problemas en el cuidado de la salud bucal. La obtención de partículas a escala atómica se logra por dos vías: reduciendo una partícula hasta lograr un tamaño nanométrico o ensamblando nanoelementos con características y propiedades específicas. Estos pequeños compuestos tienen potenciales aplicaciones en el diagnóstico, prevención y tratamiento de patologías orales desde caries, enfermedad periodontal, maloclusiones, lesiones en tejidos blandos y hasta posibles indicaciones para mejorar la estética dental y el comportamiento mecánico, biológico y óptico de materiales restauradores. Algunos desarrollos se encuentran consolidados con etapas exitosas de experimentación y aplicación clínica en tanto que otros se avizoran con expectativas de cambios revolucionarios, como aquellas relacionadas a la ingeniería tisular.

\section{Abstract}

Technological innovations in the field of Dentistry currently relate Nano-science to possible solutions to a variety of oral health problems. Nano-particles can be obtained through two methods: by reducing the size of a particle to a nonometric scale or by assembling nano-particles into one piece with specific features and properties. These compounds may have a potential use in diagnostic, prevention or treatment of oral diseases from caries, periodontal problems, malocclusion, lesions of soft tissues and even to enhance aesthetics or the mechanical, biological and optic properties of dental materials of ceramic, metallic, organic or hybrid nature. Some of these developments have already been successfully tested whereas others are expected to change dramatically the history of Dentistry such as those related to tissue engineering.

\section{Introducción:}

En 1959 el Profesor -y luego Premio Nobel de Física- Richard Feynman hace referencia pública por primera vez a las posibilidades de las nanociencias y la nanotecnología en su célebre discurso titulado "En el fondo hay espacio de sobra" (traducido del inglés "Thereisplenty of space in thebottom"). Este acontecimiento se toma actualmente como la piedra angular de los avances científicos y tecnológicos que prometen transformar en realidad la idea de "pequeñas soluciones para grandes problemas". ${ }^{1}$

De hecho, el origen del prefijo nano proviene del griego "pequeño o enano" y se aplica en términos científicos al manejo de la materia a escala subatómica, siendo un nanómetro el equivalente a la millonésima parte de un milímetro. A mayor escala, sería comparable a la relación que existe proporcionalmente entre un grano de maíz y el volumen de la esfera del planeta Tierra. 
La utilización de elementos cuyas propiedades puedan expresarse aún en tamaños ínfimos permite imaginar la posibilidad de aprovechar estos beneficios en lugares otrora inaccesibles para la reparación biológica. En el marco de la Odontología, estas innovaciones se traducen en un universo de potenciales recursos destinados al diagnóstico, prevención y tratamiento de enfermedades que se multiplican exponencialmente con los cambios demográficos de la población mundial. ${ }^{1-5}$

Para comprender mejor las dimensiones de estos avances es importante conocer qué sustancias o compuestos se pueden sintetizar a escala nanométrica, sus mecanismos de obtención y posibles aportes al equilibrio de la salud, en particular de la cavidad bucal.

\section{Nanopartículas y nanocompuestos utilizados en odontología: Métodos de síntesis u obtención}

Las tecnologías involucradas en la fabricación de nanomateriales se pueden clasificar como bottonup o top-down6. El enfoque top-down se basa en el uso de un material a granel que luego es sometido a una disminución del tamaño de partículas por debajo de la micra. Para alcanzar este objetivo se aplica energía y destacan por su uso las técnicas de homogenización de alta presión (HAP), la microfluidización y la nanomolienda asistida en medio líquido.

Por el contrario, el enfoque botton- up consiste en el "ensamblaje" de moléculas y átomos, para formar estructuras más grandes. ${ }^{7}$

La naturaleza de los materiales como así también los diferentes tipos de sistemas (nanopartículas, nanocapsulas, liposomas, nanocristales, etc.) es extensa y difícil de abordar en un artículo de este tipo, pero a tales fines se hará una descripción del estado del arte basándonos en el uso concreto de los nanomateriales en odontología y sus estrategias de obtención.

\section{Nanocristales}

Los nanocristales se obtienen utilizando procesos top-down que consumen mucha energía, y el ingrediente activo se fragmenta directamente (HAP, ultrasonido, nanomolienda asistida) en un tamaño inferior al micrométrico partiendo material a granel. Se han propuesto nanocristales de nitrato de potasio, sales de calcio pobremente solubles, fluoruro de calcio e hidroxiapatita sustituida con carbonato como sustancias activas para el tratamiento de la sensibilidad de la dentina, la remineralización de las superficies dentales y la inhibición de la caries. Estos nanocristales se pueden formular como compuestos para la higiene oral o dental, como soluciones, suspensiones, aceites, resinas u otros productos sólidos. El efecto mejorado de los nanocristales en comparación con sus polvos a granel puede explicarse por su pequeño tamaño, que les permite infiltrarse y penetrar en los túbulos dentinales o las superficies porosas de los dientes formando un depósito terapéutico. ${ }^{9-12}$

\section{Nanopartículas}

Las nanopartículas se han propuesto como sistemas de administración de fármacos para el control y la restauración de la caries, la remineralización dental, el manejo de la hipersensibilidad dentinaria, la vacuna contra la caries dental, el tratamiento dl biofilm oral, la desinfección del conducto radicuar, la anestesia local y la infección periodontal. Por ejemplo, las nanopartículas mejoran los trataientos para las enfermedades de las estructuras dentales y orales usando fármacos clásicos. Se postula que las nanopartículas se puedan administrar selectivamente a sitios o células diana. Una de las aplicaciones más importantes es el tratamiento de la enfermedad periodontal.

Comparadas con las micropartículas, las nanopartículas ofrecen ventajas, como la capacidad de penetrar áreas extracelulares e intracelulares que pueden ser inaccesibles a otros sistemas de administración debido a su pequeño tamaño, incluidas las zonas de bolsas periodontales debajo de la línea de las encías. ${ }^{13-15}$ Los estudios de microscopía confocal láser llevados a cabo por Ganem-Quintanar y col. ${ }^{16}$ establecieron que las nanopartículas biodegradables, cuando se aplican suavemente al espacio gingival porcino, pueden penetrar en las uniones estrechas del epitelio. Del mismo modo, las nanopartículas en el surco gingival podrían ser un sistema de administración de fármacos que reduzca la frecuencia de la posología, además de proporcionar una acumulación eficaz del agente activo en los sitios diana durante un período prolongado, manteniendo una tasa de liberación de fármaco efectiva en el tiempo. ${ }^{17}$

\section{Nanopartículas metálicas}

Las nanopartículas metálicas son entidades de escala submicrométrica obtenidas a partir de metales puros (por ejemplo, oro, platino, plata, titanio, zinc, cerio, hierro y talio) o sus compuestos (p. Ej., Óxidos, hidróxidos, sulfuros, fosfatos, fluoruros y cloruros $)^{8}$. Uno de los nanosistemas más documentados en odontología son las nanopartículas de plata. Debido a su pequeño 
tamaño, las nanopartículas de plata poseen una gran área de superficie disponible para la oxidación. ${ }^{18}$

La plata se ha utilizado en la purificación de agua, para curar heridas, prótesis óseas, cirugía ortopédica reconstructiva, dispositivos cardíacos, catéteres y dispositivos quirúrgicos. Los recientes avances en biotecnología han permitido la incorporación de plata ionizable en los tejidos, con el propósito de un uso clínico para reducir el riesgo de infecciones nosocomiales y también para la higiene personal. ${ }^{19}$

El efecto antimicrobiano, antifúngico y antiviral de los compuestos de plata depende de la cantidad de iones bioactivos $(\mathrm{Ag}+1)$ y su disponibilidad para interactuar con las membranas celulares bacterianas o fúngicas. ${ }^{20} \mathrm{El}$ ion de plata es biológicamente activo y puede interactuar fácilmente con proteínas, especialmente aquellas con grupos tiol, residuos de aminoácidos, aniones libres y receptores en membranas celulares de mamíferos y eucariotas. Las nanopartículas de plata, ya sea como dispersión o incorporadas en diferentes materiales, han mostrado diferentes propiedades y aplicaciones en la práctica dental como antimicrobianos, inhibidores de caries, materiales restauradores dentales, cemento de endodoncia, implantes dentales y dispositivos intraorales para prevenir la acumulación microbiana. ${ }^{18-21}$

\section{Posibles usos de la Nanotecnología en Odontología}

\section{Prevención y diagnóstico de patologías orales}

La incorporación de nanopartículas en pastas dentífricas se plantea a través de dos mecanismos: Por un lado, el modo más simple de aplicación se propone utilizando partículas nanométricas de elementos químicos con efectos antibacterianos, tales como el cinc o la clorhexidina, en cantidades suficientes para controlar el metabolismo del biofilmcariogénico y periodontopático. Por otra parte, en una propuesta de mayor complejidad se hace referencia al ensamblado de partículas de carbono entre 1 y 100 nanómetros para formar nano-robots de un diámetro promedio de 0.5 a 3 um, vehiculizados en pastas dentales (dentifrobots) y programados para interferir con exactitud a nivel celular o molecular en procesos metabólicos de la flora bacteriana ${ }^{2-4}$. Esta última hipótesis de desarrollo se asimila a la idea de una vacuna para prevención de caries, cuya dificultad se atribuye principalmente a la amplia variabilidad e inestabilidad del medio en el cual la vacuna debería efectivizar su mecanismo de acción. Estas variables podrían programarse en función de las condiciones individuales y aplicarse al diseño de los nano-robots.

En cuanto al uso de nanotecnología para el diagnóstico de patologías orales, el principio básico que se aplica a estas innovaciones implica la utilización de nano-elementos factibles de ser rastreados en órganos o sistemas. En tal sentido, dichos elementos deberán tener afinidad por las células o tejidos que se pretendan identificar, de modo tal que puedan evidenciarlos en imágenes. ${ }^{21}$

\section{Usos de la Nanotecnología en la Farmacología Odontológica.}

Es muy variada la gama de posibilidades en la utilización de productos farmacológicos ya que abarca desde el tratamiento de la hipersensibilidad hasta el transporte de sustancias terapéuticas para distintos tipos de cáncer bucal, pasando por el desarrollo de anestésicos locales que actuarían por difusión a través de los tejidos sin necesidad de agujas infiltrantes.

En el caso de los anestésicos, se están desarrollando nano-robots incorporados a sistemas coloidales que se aplicarían a nivel gingival, difundiendo rápidamente hacia los tejidos dentales, penetrando la dentina por sus túbulos hasta alcanzar la pulpa guiados por diferencias químicas y de gradientes de temperatura. Estos nano-componentes estarían programados para que el Odontólogo active su funcionamiento a través de un dispositivo, ordenando la inhibición de la conducción se sensibilidad dolorosa desde el diente durante la realización de los procedimientos, pudiendo luego revertir la situación una vez concluida la sesión. ${ }^{2-4}$

Este mecanismo también podría aplicarse para el tratamiento de la hipersensibilidad, siendo ésta una alternativa al tradicional bloqueo superficial de los túbulos dentinarios expuestos en las zonas cervicales mediante la quelación de minerales en las áreas de mayor permeabilidad.

El potencial de la Nanotecnología en el diseño de fármacos tiene centradas sus expectativas en la posibilidad de aumentar la especificidad de las drogas, reducir las dosis por una mejor solubilidad y mayor biodisponibilidad, eliminando en gran medida los efectos adversos o secundarios de los medicamentos. En esta línea existen numerosos desarrollos en etapas preliminares, siendo la terapia génica la que se avizora con excelentes perspectivas, posibilitando la reparación o el reemplazo de genes defectuosos. El sistema de distribución de genes se puede dividir en tres tipos: vectores virales, vectores no-virales y para inoculación directa de genes en determinados tejidos. ${ }^{22}$ 
De esta manera, los buenos resultados de proyectos basados en los principios mencionados podrían traducirse en avances significativos para el tratamiento químico de ciertos tipos de cáncer bucal, destruyendo las células malignas y separándolas de las sanas. ${ }^{23}$

Finalmente, en esta sección se incluye el desarrollo de nanoemulsiones para desinfección y esterilización basadas en nanopartículas de aceites que atacan y destruyen patógenos de amplio espectro, sin teñir ni manchar telas, hipoalergénicas y amigables para el ambiente. Se comercializa actualmente un producto cuyo uso se reporta para la esterilización de impresiones de pacientes protéticos.

\section{Odontología Restauradora y Cosmética}

Por mucho tiempo, la amalgama de plata fue el material de elección para restaurar la integridad anátomo-funcional de dientes afectados por el proceso de caries. A comienzos del año 2013, la Asociación Internacional de Investigación Odontológica (IADR) en conjunto con la Federación Dental Internacional (FDI) lanzaron un comunicado de prensa en el que exhortan a la comunidad científica y a los clínicos en general a elaborar una agenda para el desarrollo de nuevas alternativas al uso de la amalgama, en función del compromiso de erradicar definitivamente este material del arsenal terapéutico odontológico. En este comunicado se enumeran los requisitos que debiera reunir un material de restauración ideal: guardar una buena relación costo-eficacia, no ser tóxico, sellar la interfaz, tener la máxima estabilidad dimensional al endurecer, contribuir a la cicatrización de la cavidad generada por la lesión de caries (efecto remineralizante), ser fácil de manipular, presentar suficiente resistencia al desgaste y poder repararse. ${ }^{24}$

De los materiales disponibles en la actualidad para restauración dental encontramos los cementos, materiales del grupo de los cerámicos, cuya composición combina minerales que son activados por soluciones que favorecen la formación de sales insolubles. Estos compuestos inician su endurecimiento al mezclar la fase mineral con el activador, pudiendo éste completarse en la cavidad del diente a restaurar. Por su naturaleza inorgánica, estos materiales se integran biológicamente al elemento dentario, aunque sus propiedades físicomecánicas presentan algunas limitaciones. ${ }^{25}$ Por esta razón se han propuesto diferentes modificaciones que puedan optimizar la resistencia de los cementos al desgaste, a la fatiga, a la compresión y a la flexión, entre las cuales se incluye la incorporación de nanopartículas de plata, oro, hidroxiapatita o cinc. ${ }^{26-28}$
Por otra parte, existe otra línea de materiales restauradores que combinan los beneficios de las partículas cerámicas, pero son aglutinadas por una matriz polimérica que les otorgan plasticidad para ser modelados en la misma pieza a restaurar y luego completar su endurecimiento por óxido reducción de los monómeros. ${ }^{29}$

A diferencia de los cementos polialquenoicos que se unen químicamente al mineral del diente, las resinas compuestas necesitan de un sistema de adhesión para lograr una retención micromecánica al remanente dentario, sea a nivel del esmalte o la dentina. En tal sentido, la humectación/infiltración de las estructuras dentarias ocurre gracias a la utilización de adhesivos basados en formulaciones afines a las de la matriz orgánica de los composites. Algunas fallas adhesivas a nivel de la dentina se han atribuido a la presencia de enzimas proteolíticas, para lo cual se ha propuesto la incorporación de nanopartículas de cinc con la finalidad de neutralizar la acción de éstas. El cinc competiría con las metaloproteinasas en el sitio de unión con la molécula de colágeno que las enzimas tienden a degradar. ${ }^{30}$

De igual modo que se plantea en los cementos dentales, en las resinas compuestas también se utilizan nanopartículas para mejorar sus propiedades mecánicas y ópticas. ${ }^{31}$ De hecho, el surgimiento de los nanorrellenos corresponde en primer término a estos materiales compuestos y luego se aplicó ese principio a los cementos de ionómero vítreo a fines de la década pasada.

Las demandas de los pacientes en cuanto a la coloración del diente en las restauraciones, las preocupaciones sobre el impacto ambiental y las reacciones clínicas adversas a los materiales de relleno de amalgama, han acelerado la investigación en el desarrollo de materiales de restauración alternativos.

Uno de los avances más importantes en la tecnología de resinas compuestas fue el desarrollo de materiales basados en resinas fotocurables. Esto fue seguido por el desarrollo de un tamaño de partícula de relleno reducido y una mayor carga de estos rellenos que mejoró significativamente la aplicabilidad de este tipo de materiales.

Los "composites" de resina son ampliamente utilizados en odontología y se han convertido en uno de los materiales restauradores estéticos más comunes debido a su resistencia mecánica, excelentes propiedades estéticas, costo moderado (en comparación con la cerámica), capacidad para unir el diente, formulación mejorada, procedimientos clínicos simplificados y la disminución en el uso de amalgamas debido a la toxicidad del mercurio.

Los avances más importantes en la tecnología de resina compuesta estuvieron precipitados por la 
creciente demanda en odontología estética que ha llevado al desarrollo de compuestos de resina para restauraciones directas con propiedades físicas y mecánicas mejoradas, mejor desempeño estético y longevidad clínica. La última innovación en este campo ha sido la introducción de materiales de "nanorellenos", mediante la combinación de partículas a escala nanométrica y nanoclusters en una matriz de resina convencional. Como estos "nanofillers" no dispersan ni absorben la luz visible, proporcionan un método para dotar de opacidad al material sin interferir con su estética. También pueden permitir una alta carga, con la consecuente reducción en la contracción durante polimerización. ${ }^{31}$

Durante la última década, se ha dedicado esfuerzo al diseño de resinas "nanocompuestas" con el el foco puesto en alcanzar mayor estética, resistencia y durabilidad. Sin embargo, la investigación hasta la fecha muestra que la mayoría de los "nanorrellenos" proporcionan sólo mejoras incrementales en las propiedades mecánicas con algunas excepciones.

La nanotecnología en este punto podría ser útil además en la mejora de la adhesión de los biomateriales a los tejidos, la resistencia mecánica y la adaptación marginal, y, por lo tanto, la fiabilidad y longevidad de la restauración. Es importante destacar que los tamaños de partícula de los materiales de restauración oscilan entre 40 $\mathrm{nm}$ y $0.7 \mu \mathrm{m}$, mientras que los tamaños estructurales del cristal de hidroxiapatita, túbulo dental y varilla de esmalte se encuentran en un rango nanoscópico (1 a $10 \mathrm{~nm}$ ). Por este motivo la nanotecnología puede jugar un papel importante en la mejora de la continuidad entre la estructura dental y los biomateriales restauradores, desarrollando sistemas que formen una interfaz más estable y natural con los tejidos duros mineralizados del diente. La mejora continua en la tecnología de resinas compuestas ha dado lugar a materiales modernos con excelente durabilidad, resistencia al desgaste y estética que imitan a los dientes naturales. En particular, la incorporación de la nanotecnología en el control de la arquitectura del relleno ha logrado mejoras dramáticas en estos materiales.

Los materiales de relleno originales de resinas compuestas contenían partículas de relleno grandes de entre 10-50 $\mu \mathrm{m}$ de tamaño. Estos rellenos impartieron gran resistencia al material curado, pero debido al tamaño de los rellenos, el volumen ocupado por la resina era sustancial, lo que significa que estos materiales tendían a desgastarse rápidamente cuando se los cargaba con fuerzas oclusales y no podían pulirse con un alto brillo. Estos rellenos, que ya no se utilizan en la actualidad, eran denominados en inglés "macrofills".
Debido a lo antes expuesto, los fabricantes buscaron nuevos tipos de rellenos para lograr restauraciones con superficies altamente pulidas que imitasen mejor una superficie de esmalte. A fines de la década de 1970, se introdujeron las denominadas resinas compuestas 'microfill'. Estos compuestos utilizaron nuevos rellenos con partículas muy pequeñas de dióxido de silicio, de aproximadamente $0,04 \mu \mathrm{m}$ de diámetro. Podría decirse que estos productos fueron los primeros con "nanorellenos", aunque esa terminología todavía no se había introducido en la comunidad científica. $^{29}$

En odontología, los "nanorrellenos" podrían definirse como partículas inorgánicas con un tamaño promedio de $40 \mathrm{~nm}$. Sin embargo, la verdadera innovación no es el tamaño propiamente dicho sino la fabricación de los rellenos y la posibilidad de mejorar la carga de la fase inorgánica. El efecto de esta alta carga de relleno mejora ampliamente las propiedades mecánicas. Los compuestos "microhíbridos" con carga adicional de "nanorrellenos" son actualmente la mejor opción. Probablemente la próxima frontera de investigación en este tipo de materiales es la fabricación de un material de relleno con forma y composición que pueda imitar con mejor performance las características ópticas y mecánicas de las estructuras minerales del diente (esmalte y dentina).

Un ejemplo de estos rellenos es la sílice el cual es un material adecuado para aplicaciones biomédicas y en particular en odontología. Químicamente, la sílice es un óxido de silicio (dióxido de silicio, $\mathrm{SiO} 2$ ) que se encuentra en numerosos tejidos, incluidos huesos, tendones, aorta, hígado y riñón.

La FDA clasifica a la sílice como un agente "generalmente considerado como seguro" (GRAS), por lo que es un candidato ideal para aplicaciones biomédicas. Al ser el segundo elemento más frecuente después del oxígeno, la sílice es abundante y barata.

Durante mucho tiempo, la sílice se ha utilizado para aplicaciones dentales como un componente de relleno debido a sus propiedades físicas y ópticas. Recientemente, un producto propuesto por Müller, EGW, Wiens, M., 2012 combino hidroxiapatita y nano/microesferas de sílice) unidas por un oligopéptido que puede usarse para sellar fosas dentales, fisuras y túbulos dentinarios para prevenir la formación de caries y reducir la hipersensibilidad dentinaria.

En la última década, las nanopartículas de sílice, por ejemplo, Aerosil (Degussa), HDK (Wacker), Cab-O-Sil (Cabot Corp.) se han propuesto como rellenos a nanoescala para mejorar las resinas dentales. Estas partículas pueden mejorar el comportamiento reológico, la resistencia al 
rayado/abrasión y la dureza superficial de los productos finales.

Se considera también la inclusión de nanopartículas de zafiro y diamante en matrices resinosas para el tratamiento superficial cosmético del esmalte. Sin embargo, no existen aún productos que se comercialicen con estas características.

\section{Ingeniería tisular}

La meta soñada por quienes trabajan en la fabricación de tejidos orales es sin duda la posibilidad de cultivar células in-vitro o en un reborde alveolar humano a partir de las cuales crezca un nuevo diente que reemplace el elemento perdido. La regeneración total de una pieza dentaria a partir de las conocidas células madre es el desarrollo que ocupa predominantemente a la ingeniería tisular.

En relación a la denominada renaturalización dentaria, el trasporte de factores de crecimiento, minerales y elementos antibacterianos a zonas del diente para intentar recuperarlo hasta la restitución de su integridad anatómica y funcional, sería una alternativa más biológica para las restauraciones dentarias como consecuencia de lesiones traumáticas o por caries. ${ }^{32}$ Es posible vincular estos desarrollos con algunos avances en el campo de la Endodoncia, en los procesos de revascularización pulpar, a partir de la cual se logra regenerar el tejido en dientes con procesos necróticos. ${ }^{33}$

Por otra parte, la ingeniería tisular se vincula con la Nanotecnología para la síntesis de minerales que promuevan la regeneración de defectos óseos o la inducción de la neoformación ósea en zonas donde luego se procederá a la instalación de implantes dentales.3-4 Cabe mencionar que también se investiga la optimización de la oseointegración mediante el tratamiento superficial de los implantes con coberturas nanoparticuladas que faciliten depósitos de células óseas y prevengan las peri-implantitis. ${ }^{34}$

\section{Conclusión:}

La Nanotecnología abre infinitas posibilidades de investigación y desarrollo de nuevas plataformas destinadas al diagnóstico, prevención y tratamiento de las patologías orales. El conocimiento de sus fundamentos y potencialidades permitirá a la ciencia odontológica avanzar en una profundización de los paradigmas basados en la máxima conservación de tejidos y la implementación de estrategias simples, efectivas y de alcance masivo a nivel comunitario.

\section{Agradecimientos:}

Para la elaboración del presente artículo se recibió la colaboración de los estudiantes de la Carrera de Odontología, Facultad de Ciencias de la Salud, Universidad Católica de Córdoba, Ángel Gabriel Torres, Josefina Taborda, Ana Solange Bilinksi, Sabrina Argüello, Paula Angelaccio y Doriana Torres, quienes aportaron información recopilada en búsqueda bibliográfica.

\section{Bibliografía:}

1. Cantín M, Vilos C, Suazo I. Nanoodontología: el Futuro de Odontología Basada en Sistemas Nanotecnológicos. Int J Odontostomat, 4(2):127-132, 2010. http://dx.doi.org/10.4067/S0718381X2010000200005

2. Nagpal A, Kaur J, Sharma S, BansalA, Sachdev P. Nanotechnology-the Era of MolecularDentistry. Indian J DentSci 2011;3: 80-2.

3. Freitas RA Jr. Nanodentistry. J Am DentAssoc 2000;131(11):1559-65.

4. Kanaparthy R, Kanaparthy A. Thechangingface of dentistry: nanotechnology. Int $\mathbf{J}$ Nanomed 2011;6;2799-804.

5. Chen F, Cai W, eds. HybridNanomaterials. Design, Synthesis, and BiomedicalApplications, CRC Press, Taylor and Francis Group, Boca Raton, Florida, 2017.

6. Silva GA. Neurosciencena notechnology: progress, opportunities and challenges. NatRevNeurosci. 2006; 7:65-74.

7. Zhang S. Fabrication of novel biomaterialsthrough molecular self-assembly. NatBiotechnol. 2003; 21:1171-1178.

8. Mihranyan A, Ferraz N, Strømme M. Current status and futureprospects of nanotechnology in cosmetics. Prog Mater Sci 2012,57:875910.

9. Gazzaniga G, RoveriN, Rimondini L, Palazzo B, Lafisco M. Biologically active nanoparticles of a carbonatesubstitutedhydroxyapatitteprocessfortheirpre paration and composition sincorporating thesame. 2007 WO137606A1.

10. Henkel AAK, Suthech GMBH Co. KG, Barth A, Kropf C, Poth T. Oral and dental careproduct. 2005, WO020878A2. 
11. Chen FM, An Y, Zhang M. New insightsinto and novel applications of releas etechnologyfor periodontal reconstructive therapies. J Control Release 2011,149:92-110.

12. Xu HHK, Weir MD, Sun L, Moreau JL, Takagi S, Chow LC, Antonucci JM. Strongnano compositeswith $\mathrm{Ca}, \mathrm{PO} 4$, and $\mathrm{F}$ releasefor caries inhibition. J Dent Res 2010,89:19-28.

13. Jain N, Jain GK, Javed S, Iqbal Z, Talegaonkar S, Ahmad FJ, Khar RK. Recentapproachesforthetreatment of periodontitis. DrugDiscovToday 2008,13:932-943.

14. Kashi TSJ, Eskandarion S, EsfandyariManesh M, Marashi SMA, Samadi N, Fatemi SM, Atyabi F, Eshraghi S, Dinarvand R. Improveddrugloading and antibacterialactivity of minocycline-loaded PLGA

nanoparticlespreparedbysolid/oil/water ion pairingmethod. Int J Nanomed 2012,7:221234.

15. Bhardwaj A, Bhardwaj, SV, Pandey R. Advances in periodontal drugdeliverysystems.Int J Novel DrugDelivTech 2012,2:271-275.

16. Ganem-Quintanar A. Étude de la permeábilité de la muqueuseorale: évaluation de différentesapproachespouraugmenter le passage des principesactifs, Ph.D. thesis, Universite' de Geneve, Universite' Claude Bernard, Lyon, Genéve, 1997.

17. Kong, L.X., Peng, Z. Li, S.D., Bartold, P.M. Nanotechnology and its role in themanagement of periodontal diseases. Periodontology2006; 40:184-196.

18. García-Contreras R, Argueta-Figueroa L, Mejía-Ruvalcava C, Jiménez-Martínez R, Cuevas Guajardo S, Sánchez-Reyna PA, Mendieta-Zeron H. Perspectivesforthe use of silvernanoparticles in dental practice. IntDent J 2011,61:297-301.

19. Monteiro DR, Gorup LF, TakamiyaAS, Rodríguez de Camargo E, FilhoACR, Barbosa DB. Silver distribution and reléase from anntimicrobialdenture base resincontainingsilverlloidalnanoparticles. $\mathbf{J}$ Prosthodont 2012,21:7-15.

20. Lansdown AB. Silver 2: Toxicity in mammals and howitsproductsaidwoundrepair. J Wound Care 2002,11:173 -177.
21. Lansdown AB. Silver I: Itsantibacterialproperties and mechanism of action. J WoundCare 2002,11:125 - 130.

22. Jain KK. Nanodiagnostics: application of nanotechnology in molecular diagnostics. ExpertRev Mol Diagn2003; 3:153 - 61.

23. Goverdhana S, Puntel M, Xiong W, Zirger JM, Barcia C, Curtin JF, et al. Regulable gene expressionsystemsfor gene therapyapplications: progress and futurechallenges. Mol Ther 2005;12(2):189211.

24. International Associationfor Dental Research. Launching a dental materialsresearch agenda [Internet]. Alexandria: TheAssociation; 2012 [cited 2013 Jan 14]. Availablefrom: http://www.iadr.org/files/public/13DMIW_S ummary.pdf

25. Sidhu SK, Nicholson JW. A Review of GlassIonomerCementsforClinicalDentistry. J FunctBiomater. 2016 Jun 28;7(3). pii: E16. doi: 10.3390/jfb7030016.

26. Baig MS, Fleming GJ. Conventionalglassionomermaterials: A review of thedevelopments in glasspowder, polyacidliquid and thestrategies of reinforcement. J Dent. 2015, Aug;43(8):897912. doi: 10.1016/j.jdent.2015.04.004.

27. Molina GF, Cabral RJ, Mazzola I, Brain L, Frencken JE. Mechanical performance of encapsulatedrestorativeglass-

ionomercementsfor use withAtraumaticRestorativeTreatment (ART). J Appl Oral Sci 2013; 21(3):243-249.

28. Najeeb S, Khurshid Z, Zafar MS, Khan AS, Zohaib S, Martí JM, Sauro S, Matinlinna JP, Rehman IU. Modifications in Glass Ionomer Cements: Nano-SizedFillers and BioactiveNanoceramics. Int J Mol Sci. 2016 Jul 14;17(7). pii: E1134. doi: 10.3390/ijms17071134.

29. Beun S, Glorieux T, Devaux J, Vreven J, Leloup G. Characterization of nanofilledcompared to universal and microfilledcomposites. Dent Mater 2007;23(1):51-9.

30. Osorio R, Cabello I, Medina-Castillo AL, Osorio E, Toledano M. Zincmodifiednanopolymersimprovethequality of resin-dentinbonded interfaces. Clin Oral Investig. 2016 Dec;20(9):2411-2420. doi: 10.1007/s00784-016-1738-y

31. Vollath D. Nanoparticles - Nanocomposites Nanomaterials. AnIntroductionforBeginners, 
WILEYVCH Verlag GmbH\& Co, Weinheim, Germany, 2013.

32. Chen HF, Clarkson BH, Sunk, Mansfield JF. Selfassembly synthetichydroxyapatitenanorodsintoenamelp rismlikestructure. J Colloid Interface Sci 2005;288(1):97-103.

33. Lin LM, Ricucci D, Huang GT. Regeneration of thedentinepulpcomplexwithrevitalization/revascularizat iontherapy: challenges and hopes. IntEndod J. 2014 Aug;47(8):713-24. doi: 10.1111/iej.12210.

34. Le Gu'ehennec L, Soueidan A, Layrolle P, Amouriq Y. Surface treatments of titanium dental implants forrapidosseointegration, Dent Mater 2007;23(7):844-54.

\section{Palabras claves:}

NANOTECNOLOGÍA, MATERIALES DENTALES, INNOVACIONES TECNOLÓGICAS.

\section{Keywords:}

NANOTECHNOLOGY, DENTAL

MATERIALS, TECHNOLOGICALINNOVATIONS.

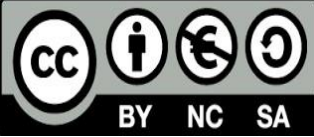

